\title{
The impact of medium dose UVA1 phototherapy on pruritus, DLQI and SCORAD index in patients with atopic dermatitis
}

\author{
Karolina Malinowska, Anna Woźniacka, Jarosław Bogaczewicz \\ Department of Dermatology and Venereology, Medical University of Lodz, Lodz, Poland \\ Adv Dermatol Allergol 2020; XXXVII (6): 962-967 \\ DOI: https://doi.org/10.5114/ada.2019.88465
}

\begin{abstract}
Introduction: Atopic dermatitis (AD) is featured by pruritus, which causes diminished quality of life. Little clinical data exists concerning the use, efficacy and side effects of UVA1 phototherapy in AD patients.

Aim: To determine the effectiveness of medium-dose UVA1 phototherapy in AD treatment.

Material and methods: Thirty-six patients with AD were irradiated with medium-dose UVA1 $\left(45 \mathrm{~J} / \mathrm{cm}^{2}\right)$ as monotherapy for 4 weeks for a total of 20 sessions (daily irradiations during weekdays only). Clinical status was evaluated with the visual analogue scale for pruritus, Dermatology Life Quality Index (DLQI) for evaluating general well-being and the SCORAD index. All parameters were measured twice: before and after phototherapy.

Results: UVA1 phototherapy resulted in a significant $(p<0.001)$ decrease in pruritus, improvement in DLQI $(p<0.001)$ and SCORAD $(p<0.001)$. Before phototherapy, the intensity of pruritus and SCORAD index correlated with DLQI ( $r=0.34, p<0.05$ and $r=0.61, p<0.05$, respectively). Similarly, after irradiation, pruritus correlated with DLQI, and SCORAD index correlated with DLQI $(r=0.51, p<0.05$ and $r=0.55, p<0.05$, respectively). No severe adverse effects were noted during the study.

Conclusions: Phototherapy with medium-dose UVA1 irradiation exerts a significant antipruritic effect, decreases the severity of the disease and improves the quality of life of AD patients. This technique can therefore be used as a safe and effective treatment method.
\end{abstract}

Key words: atopic dermatitis, phototherapy, UVA1, pruritus, itch, Dermatology Life Quality Index.

\section{Introduction}

Light has been known to have a beneficial effect on the human organism for centuries. Nowadays, phototherapy is typically associated with doses of UVA, i.e. UVA1. UVA1 phototherapy is a newer approach that uses only the longer, non-erythemogenic UV wavelengths (340 to $400 \mathrm{~nm}$ ), thus avoiding the risk of sunburn reactions associated with the shorter-wavelength UVA2 (320 to $340 \mathrm{~nm}$ ) and UVB (290 to $320 \mathrm{~nm}$ ). The first clinical observations on the effectiveness of UVA1 radiation concerned patients suffering from atopic dermatitis (AD). This disease is a chronic, pruritic and relapsing inflammatory dermatosis, characterized by typical localization and specific skin lesions [1]. Pruritus is a hallmark of AD, and a key criterion in the diagnostic criteria proposed by Hanifin and Rajka [2]. Atopic dermatitis may coexist with other atopic diseases, such as asthma, allergic rhinitis and allergic gastritis [1]; its symptoms detract from the quality of life in $A D$ patients, and its chronic and relapsing course represents an ongoing problem in their daily life [3]. UVA1 has a greater therapeutic effect than other UV wavelengths as it can penetrate more deeply into the dermis than UVB, thus targeting cells that reside in or infiltrate the dermis, such as dendritic cells, fibroblasts, mast cells, and T and B lymphocytes. UVA1 phototherapy is believed to act by inducing apoptosis through the action of active oxygen molecules, such as singlet oxygen, hydrogen peroxide or superoxide radicals, and to activate programmed and non-programmed cell death. Its effectiveness is similar to that of photochemotherapy, i.e. the combination of psoralens with a wide spectrum of UVA radiation (PUVA) [4-8]. UVA1 radiation induces the production of inflammatory mediators and modulates the expression of superficial zone proteins $[8,9]$. The first clinical observations on effectiveness of UVA1 radiation were obtained from patients suffering from $A D$, which

Address for correspondence: Jaroslaw Bogaczewicz MD, PhD, Department of Dermatology and Venereology, Medical University of Lodz, 1 Hallera St, 90-647 Lodz, Poland, phone: +48 4263930 95, fax: +48 42688 45 65, email: jaroslaw.bogaczewicz@umed.lodz.pl Received: 11.06.2019, accepted: 16.06.2019. 
found high-dose UVA1 phototherapy $\left(130 \mathrm{~J} / \mathrm{cm}^{2}\right)$ to have beneficial results on patients with $A D$ exacerbation [9].

\section{Aim}

The aim of the present study was to determine the effectiveness of medium-dose UVA phototherapy in reducing pruritus, and improving quality of life and SCORAD in patients with atopic dermatitis.

\section{Material and methods}

The study comprised a limited number of 36 patients with atopic dermatitis, including 20 women and 16 men aged 17 to 61, mean $30.8 \pm 11.3$ years. The diagnosis was established according to Hanifin and Rajka [2]. The study was approved by the local Ethics Committee (RNN/16/14/ KE) and in accordance with the Helsinki Declaration of 1975, as revised in 2000 and the informed consent was obtained from all participants prior to the study. The Dermatology Life Quality Index (DLQI) was used to assess the impact of AD on the lives of the patients [10]. A visual analogue scale was used to assess pruritus, in which 0 indicates its absence and 10 the most extreme form. An objective clinical estimation was performed of atopic dermatitis; to help minimize bias, only one person (K.M.) calculated the SCORAD index (a clinical tool for assessing the severity, i.e., extent and intensity of $A D$ ), and this evaluator was unaware of the results of the molecular studies. The pruritus, DLQI and SCORAD assessments were performed twice, i.e. before (baseline assessment) and after UVA1 phototherapy [11]. Patients with the photosensitive form of $A D$ and with concomitant or history of neoplastic diseases were excluded from the study.

Medium doses of UVA1 phototherapy were used as monotherapy in all patients: Gambichler et al. define medium doses of UVA1 as those ranging from 40 to $80 \mathrm{~J} /$ $\mathrm{cm}^{2}$ [12]. Topical management was restricted to the use of emollients. In the present study, Medisun 24000 (Schulze \& Bohm, Gmbh, Germany) was used and the protocol of UVA1 irradiation comprised one exposure daily, on weekdays from Monday to Friday, ramping from a starting dose of $10 \mathrm{~J} / \mathrm{cm}^{2}$ through 20 and 30 to a maximum $45 \mathrm{~J} / \mathrm{cm}^{2}$. The final dose of $45 \mathrm{~J} / \mathrm{cm}^{2}$ was maintained for up to 20 days. In patients with AD no systemic drugs were used, and topical management was restricted to the use of emollients.

Informed consent was obtained prior to the study from all participants; the study was approved by the local ethics committee (RNN/16/14/KE).

\section{Statistical analysis}

Statistical analysis was performed using Statistica software, version 13.0 (Statsoft, Poland). The Wilcoxon signed-rank test was used to compare two repeated measurements in a single sample of AD patients before and after UVA1 phototherapy. The Spearman's rank cor- relation coefficient was used to evaluate the relationship between two variables, and the correlation was displayed graphically by scatterplot. Results were represented as median with lower $\left(25^{\text {th }}\right)$ and upper $\left(75^{\text {th }}\right)$ quartile $\left(25^{\text {th }}\right.$ $75^{\text {th }}$ centile). The descriptive data regarding age were displayed as mean \pm standard deviation of the mean. In all calculations, a $p$-value less than 0.05 was regarded as statistically significant.

\section{Results}

All analyzed parameters, i.e. pruritus, DLQI and SCORAD decreased significantly after irradiation. Before UVA1 phototherapy, DLQI correlated with the level of pruritus $(r=0.34, p<0.05)$ and the SCORAD index $(r=0.61, p<$ 0.05 ) in patients with AD (Figures 1 and 2).

UVA1 phototherapy resulted in a significant ( $p<$ $0.001)$ decrease in pruritus: the median value fell from eight points at baseline to three points after treatment (Figure 3).

After UVA1 phototherapy, a significant improvement in DLQI was found. The median level decreased from 15 to 6 points $(p<0.001)$ (Figure 4$)$.

UVA1 phototherapy resulted in a significant improvement in SCORAD index in comparison to the baseline assessment. In patients with atopic dermatitis, median SCORAD decreased from 61.4 to 32.3 points $(p<0.001)$ (Figure 5).

After medium-dose UVA1 phototherapy, DLQI correlated with the degree of pruritus $(r=0.51, p<0.05)$ and SCORAD index $(r=0.55, p<0.05)$ (Figure $6 \mathrm{~A}, \mathrm{~B})$.

The irradiations were generally well tolerated. Only 5 out of 36 patients reported light intensity and transient skin burning and redness. These sensations did not result in the withdrawal from UVA1 irradiation for any patient.

\section{Discussion}

It is generally accepted that cases of AD are associated with a T-cell mediated immune response against allergens. The majority of clinical, histological, and immunohistochemical features of AD resemble allergic contact dermatitis. The cytokines produced by $T$ cells contribute to the provocation and maintenance of skin lesions. UVA1 radiation is believed to activate alloreactive T cells by the abrogation of epidermal Langerhans cell function and this process may be responsible for the therapeutic effectiveness of UVA1 irradiation in the management of AD patients.

The AD patients report that their skin condition has significant negative effects on their quality of life, and these correlate with severity and unequal control of the disease [13]. The most intrusive symptom of AD is itching, and this is generated by the direct stimulation of nerve fibers occurring as a result of impairments in the skin barrier as well as the action of cytokines and neuro- 


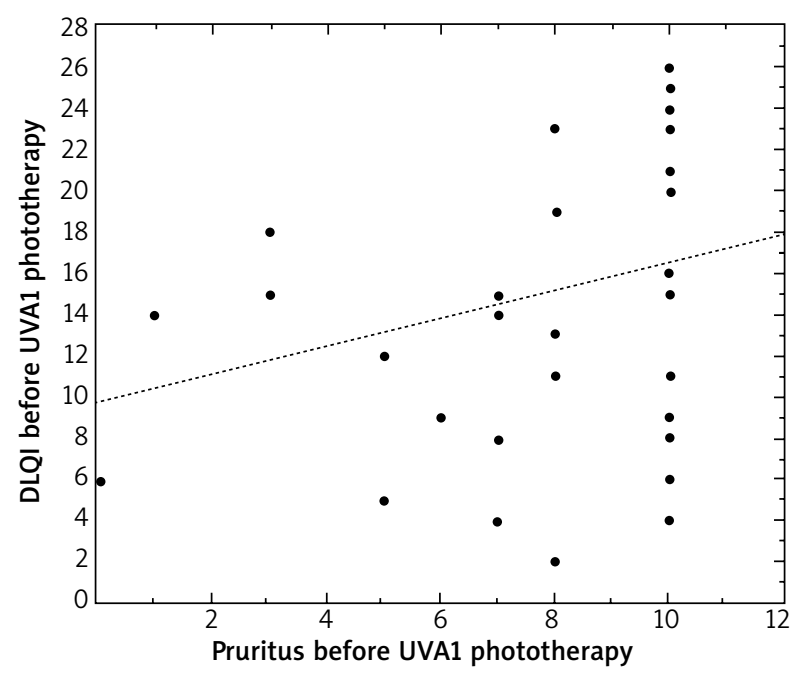

Figure 1. Correlation between the level of pruritus and DLQI in patients with atopic dermatitis before UVA1 phototherapy

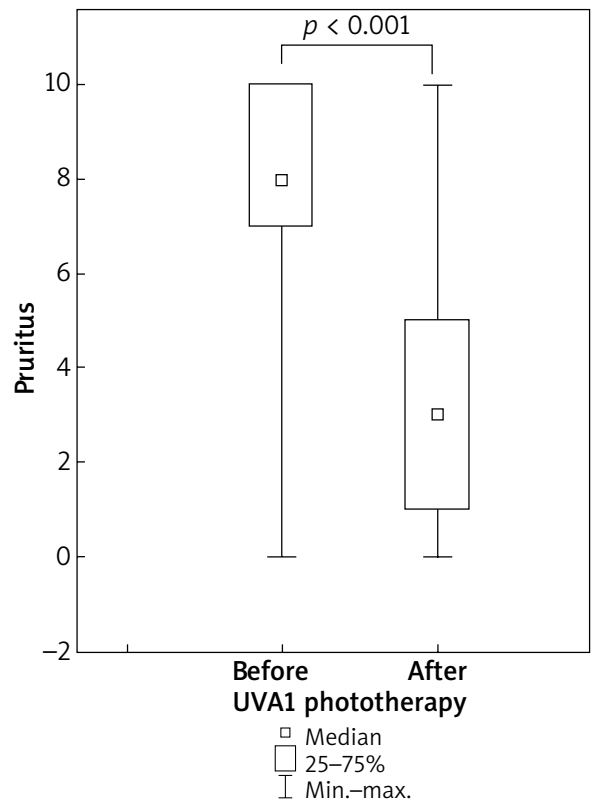

Figure 3. Decrease in pruritus in patients with atopic dermatitis undergoing medium-dose UVA1 phototherapy

peptides released by inflammatory and resident cells [13, 14]. A range of immunomodulating mediators and their receptors are involved in pruritus [15].

Therefore, the present study was performed to determine the effectiveness of medium-dose UVA1 phototherapy in reducing pruritus, and improving the quality of life and SCORAD in patients with atopic dermatitis. An important finding was that UVA1 irradiation resulted in a significant improvement in skin lesions, pruritus and Dermatology Life Quality Index (DLQI). Although pruritus

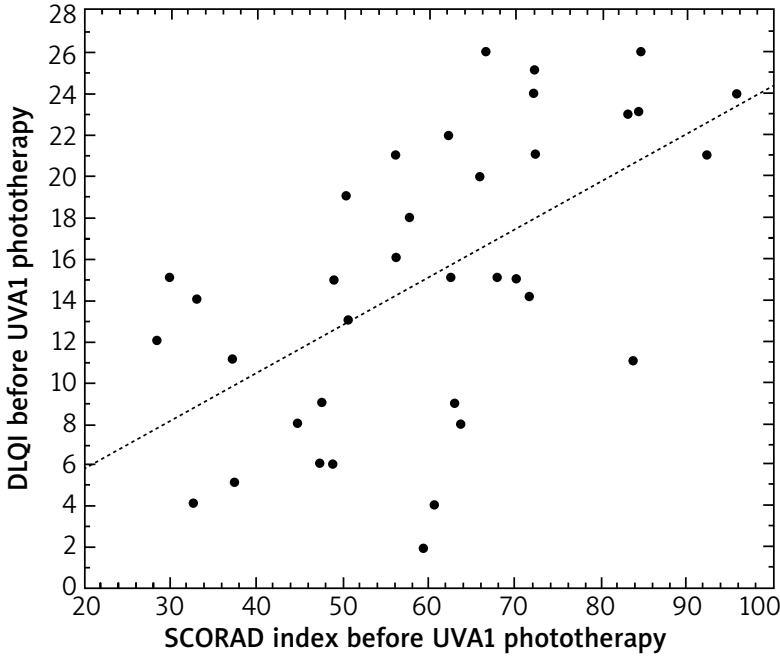

Figure 2. Correlation between the SCORAD index and DLQI in patients with atopic dermatitis before UVA1 phototherapy

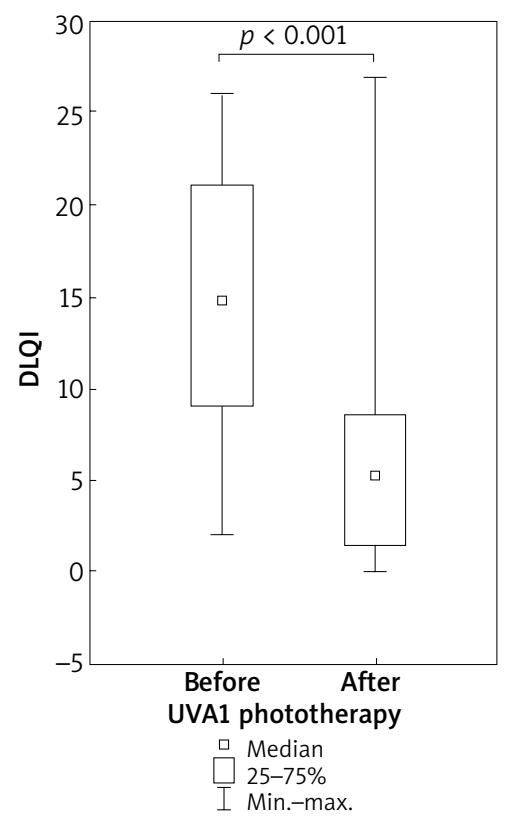

Figure 4. Improvement in DLQI in patients with atopic dermatitis undergoing medium-dose UVA1 phototherapy

is the main symptom of $A D$, and has a high impact on quality of life [16], its pathophysiology is still poorly understood; however, itching is known to be excited by the action of neuropeptides on unmyelinated nerve fibers in the papillary dermis and epidermis [17]. As a great deal of cytokines, neuropeptides, proteases and other mediators are involved in the itch sensation $[18,19]$, it is likely that UVA1 phototherapy exerts its antipruritic effect by inhibiting the expression of cytokines and chemokines in the epidermis and dermis: UV light is known to interfere 
with the structures and mediators involved in the induction and perception of pruritus [20]. Gambichler et al. report that UVA1 radiation has a significant influence at the genetic level on the expression of cytokines involved in the pathomechanism of AD. Patients with exacerbation of $A D$ were irradiated with a dose of $50 \mathrm{~J} / \mathrm{cm}^{2}$ for 6 weeks (cumulative dose $900 \mathrm{~J} / \mathrm{cm}^{2}$ ). After treatment, a considerable decrease in interleukin IL-5, IL-13 and IL-31 mRNA expression was observed. The results correlated with a clinical improvement in skin condition and a reduction in itching [21]. In various skin diseases of chronic pruritus, including prurigo, phototherapy is an effective form of treatment $[22,23]$. Consecutive reports confirm that UVA1 irradiation displays good effectiveness: highand medium dose UVA1 treatment was found to alleviate pruritus among patients with urticaria pigmentosa [24].

UVA1 therapy appears not only effective but also safe: only 5 of the 36 patients tested in the present group reported transient and not intense sensations, such as skin burning and redness, following treatment. It may serve as a recommended scheme in treating AD.

The clinical efficacy of UVA1 in AD is believed to derive from its potential to decrease the expression of proinflammatory cytokines at the mRNA level, and reduce IL-31 production by T cells and granulocytes such as eosinophils and mast cells [21, 25]. UVA1 exposure induces apoptosis in infiltrating T cells, which have been suggested to play a major role in pruritus induction by the production and release of cytokines [21]. Interleukin IL-31 binds to a receptor on peripheral nerve fibers, and has been shown to link inflammation with pruritus in the skin, with recent studies demonstrating that IL-31 mRNA expression is present in skin samples of inflammatory diseases, such as AD and allergic contact dermatitis [13]. IL-31 plays a major part in causation of pruritus and worsening of the course in $\operatorname{AD}[26,27]$, and

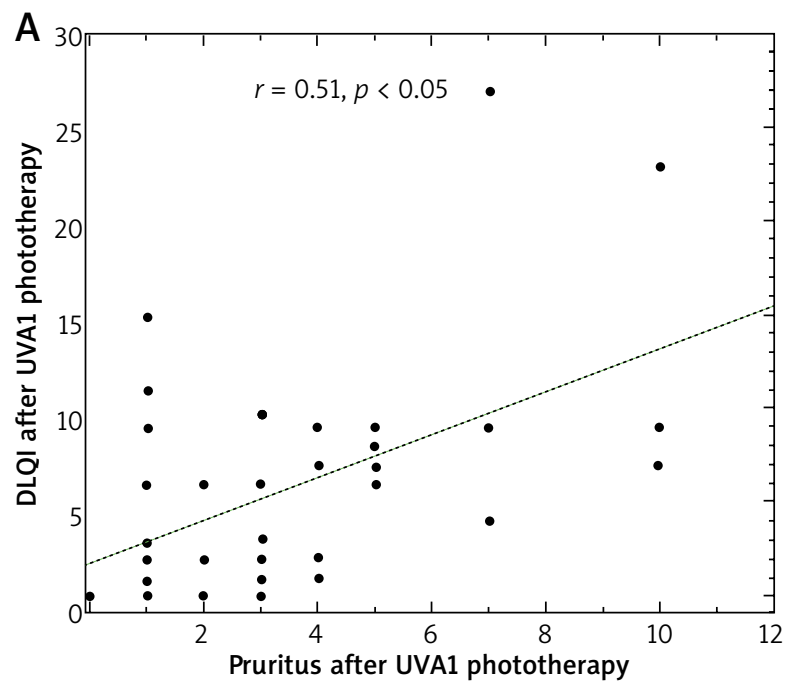

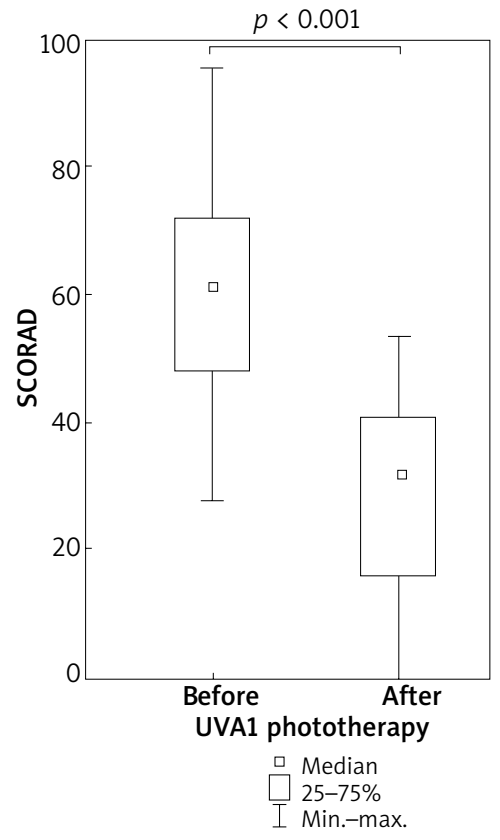

Figure 5. Improvement in SCORAD index in patients with atopic dermatitis undergoing medium dose UVA1 phototherapy

it is significantly overexpressed in pruritic atopy compared with non-pruritic psoriatic skin inflammation [25]. Subcutaneous, intradermal or intravenous administration of IL-31 into normal mice induces severe pruritus [28]. Prick testing with an IL-31 solution triggers the itch sensation in humans, and it has been demonstrated that blockade of IL-31 signals by a specific antibody reduces pruritus in patients with $A D$ [28]. IL-31 receptor $\alpha$ protein has also been detected in the nerve fibers of the dermis of AD patients and in neurons in normal human dorsal root ganglia.

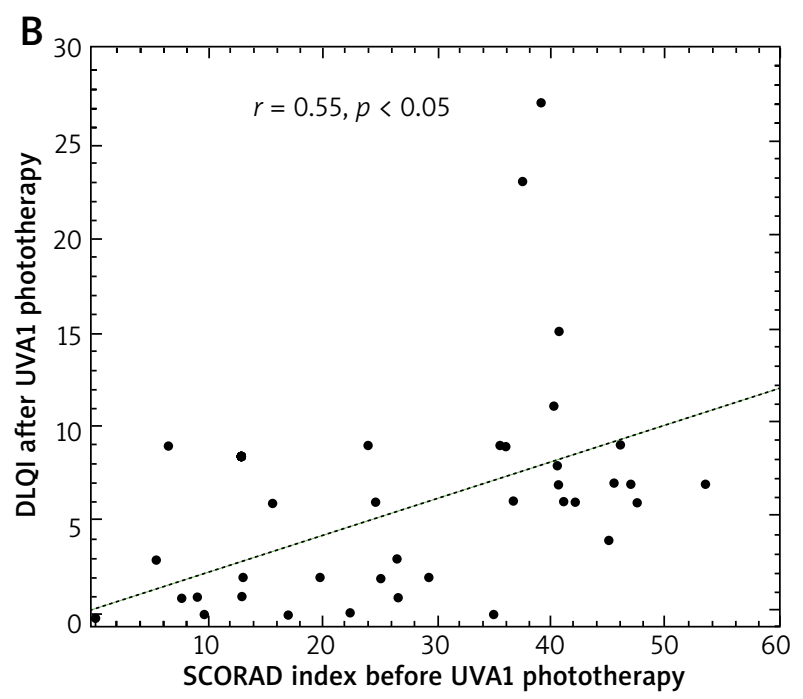

Figure 6. Correlations between the level of pruritus and DLQI (A) and between the SCORAD index and DLQI (B) in patients in patients with atopic dermatitis after medium-dose UVA1 phototherapy 
The sensation of itching is directly mediated by unmyelinated C fibers of primary sensory neurons of dorsal root ganglia. Some reports suggest that IL-31 might induce pruritus by direct modulation of the function of sensory neurons, leading to the outgrowth of primary small-diameter dorsal root ganglia [29]. Interleukin IL-31 may induce a pruritic sensation by activating the IL-31 receptor $\alpha$ protein expressed by keratinocytes; this in turn may induce keratinocyte-derived mediators, such as thymic stromal lymphoprotein, which activate unmyelinated C fibers in the skin [17].

Chronic pruritus can dramatically reduce quality of life among patients. The Dermatology Life Questionnaire Index (DLQI) was created to reflect the subjective impact of skin disease in clinical Dermatology practice; it is widely used to assess quality of life among patients with pruritic conditions of cutaneous origins [30]. The DLQI was designed in 1994 [10]. Significant improvements in DLQI score have previously been observed after phototherapy in patients with $A D$ [30]. In the present treatment group, medium-dose UVA1 treatment was found to be associated with a reduction in SCORAD index, decrease of pruritus and improvement of DLQI. Successive studies have also found medium-dose UVA1 $\left(45 \mathrm{~J} / \mathrm{cm}^{2}\right)$ to have a beneficial effect in the course of AD [4].

Our findings confirm those from introductory reports, which highlight the efficacy of UVA1 phototherapy in the treatment of AD [4-8]. Nevertheless, the pathophysiology of pruritus and the possible mechanisms by which UV radiation has its antipruritic effect remain unexplained, and future clinical investigations are needed. The clinical efficacy of UVA1 in AD is believed to result from a decrease in the levels of various proinflammatory mediators. UVA1 radiation appears to act by inducing apoptosis among the cells of the dermis, such as CD4 lymphocytes, mastocytes and granulocytes, as well as fibroblasts, dendritic and other infiltrating inflammatory cells.

\section{Conclusions}

Medium-dose UVA1 phototherapy has significant antipruritic effects, and improves atopic dermatitis, expressed by the SCORAD index, and improves quality of life, indicated by the DLQI questionnaire. It is a safe, effective and recommended method of treatment in $A D$ patients.

\section{Acknowledgments}

This study was supported by a grant for scientific purposes (No. 503/1-152-01/503-11-255) from the Medical University of Lodz, Poland.

\section{Conflict of interest}

The authors declare no conflict of interest.

\section{References}

1. Nowicki R, Trzeciak M, Wilkowska A, et al. Atopic dermatitis: current treatment guidelines. Statement of the experts of the Dermatological Section, Polish Society of Allergology, and the Allergology Section, Polish Society of Dermatology. Adv Dermatol Allergol 2015; 32: 239-49.

2. Hanifin JM, Rajka G. Diagnostic features of atopic dermatitis. Acta Derm Venereol (Stockholm) 1980; 92: 44-7.

3. Silverberg JI, Gelfand JM, Margolis DJ, et al. Severity strata for POEM, PO-SCORAD, and DLQI in US adults with atopic dermatitis. Ann Allergy Asthma Immunol 2018; 121: 464-8.

4. Marloes-Polderman CA, Wintzen M, Cessie S, et al. UVA-1 cold light therapy in the treatment of atopic dermatitis: 61 patients treated in the Leiden University Medical Center. Photodermatol Photoimmunol Photomed 2005; 21: 6-93.

5. Godar ED. UVA1 radiation triggers two different final apoptotic pathways. I Invest Dermatol 1999; 112: 3-12.

6. Krutmann J, Morita A. Mechanisms of ultraviolet (UV) B and UVA phototherapy. I Investig Dermatol Symp Proc 1999; 4: 2-70.

7. Plettenberg H, Stege H, Megahed M. Ultraviolet A1 (340-400 $\mathrm{nm}$ ) phototherapy for cutaneous T-cell lymphoma. J Am Acad Dermatol 1999; 41: 47-50.

8. Malinowska K, Sysa-Jędrzejowska A, Woźniacka A. UVA1 phototherapy in dermatological treatment. Adv Dermatol Allergol 2011; 28: 53-8.

9. Krutmann J, Schopf E. High dose UVA1 phototherapy: a novel and highly effective approach for the treatment of acute exacerbation of atopic dermatitis. Acta Derm Venerol Suppl (Stockh) 1992; 176: 2-120.

10. Finlay AY, Khan GK. Dermatology Life Quality Index (DLQI)a simple practical measure for routine clinical use. Clin Exp Dermatol 1994; 19: 210-6.

11. Kunz B, Oranje AP, Labrèze L, et al. Clinical validation and guidelines for the SCORAD index: consensus report of the European Task Force on Atopic Dermatitis. Dermatology 1997; 195: 9-10.

12. Gamblichler T, Terras S, Kreuter A. Treatment regimes, protocols, dosage, and indications for UVA1 phototherapy: facts and contorversies. Clin Dermatol 2013; 31: 438-54.

13. Simon D, Wollenberg A, Renz H, et al. Atopic dermatitis: Collegium Internationale Allergologicum (CIA) update 2019. Int Arch Allergy Immunol 2019; 178: 207-18.

14. Meng J, Steinchoff M. Molecular mehanisms of pruritus. Curr Res Transl Med 2016; 64: 203-6.

15. Rossbach K, Nassenstein C, Gschwandtner M, et al. Histamine $\mathrm{H} 1, \mathrm{H} 3$, and $\mathrm{H} 4$ receptors are involved in pruritus. Neuroscience 2011; 190: 89-102.

16. Finlay AY. Quality of life in atopic dermatitis. J Am Acad Dermatol 2001: 45: 64-6.

17. Kato A, Fujii E, Watanabe T, et al. Distribution of IL-31 and its receptor expressing cells in skin of atopic dermatitis. J Dermatol Sci 2014; 74: 229-35.

18. Steinhoff M, Bienenstock J, Schmelz M, et al. Neurophysiological, neuroimmunological, and neuroendocrine basis of pruritus. J Invest Dermatol 2006; 126: 1705-18.

19. Wilson SR, Thé L, Batia LM, et al. The epithelial cell-derived atopic dermatitis cytokine TSLP activates neurons to induce itch. Cell 2013; 155: 285-95.

20.Legat FJ. The antipruritic effect of phototherapy. Front Med 2018; 5: 333

21. Gambichler T, Kreuter A, Tomi NS, et al. Gene expression of cytokines in atopic eczema before and after ultraviolet A1 phototherapy. Br J Dermatol 2008; 158: 1117-20. 
22. Rivard J, Lim HW. Ultraviolet phototherapy for pruritus. Dermatol Ther 2005; 18: 344-54.

23. Nakamura M, Koo JYM. Phototherapy for the treatment of prurigo nodularis: a review. Dermatol Online J 2016; 22: pii: 13030/qt4b07778z.

24.Gobello T, Mazzanti C, Sordi D, et al. Medium- versus highdose ultraviolet $\mathrm{A} 1$ therapy for urticaria pigmentosa: a pilot study. J Am Acad Dermatol 2003; 49: 679-84.

25. Sonokoly E, Muller A, Laurema A. IL-31: a new link beetwen T cells and pruritus in atopic skin infammation. J Allergy Clin Immunol 2006; 117: 411-7.

26. Baron JM, Lüscher B. IL-31 expression by inflammatory cells is preferentially elevated in atopic dermatitis. Acta Derm Venereol 2012; 92: 5-6.

27. Gangemi S, Quartuccio S, Casciaro M, et al. Interleukin 31 and skin diseases: a systematic review. Allergy Asthma Proc 2017; 38: 401-8.

28. Furue M, Yamamura K, Kido-Nakahara M, et al. Emerging role of interleukin-31 and interleukin-31 receptor in pruritus in atopic dermatitis. Allergy 2018; 73: 29-36.

29. Feld M, Garcia R, Buddenkotte J, et al. The pruritus- and Th2-associated cytokine IL-31 promotes growth of sensory nerves. J Allergy Clin Immunol 2016; 138: 500-8.

30.Väkevä L, Niemelä S, Lauha M, et al. Narrowband ultraviolet B phototherapy improves quality of life of psoriasis and atopic dermatitis patients up to three months: results from an observational multi-center study. Photodermatol Photoimmunol Photomed 2019, doi: 10.1111/phpp.12479. Epub ahead of print. 